\title{
Die Bedeutung der familiären Lernumwelt für Vorläufer schriftsprachlicher Kompetenzen im Vorschulalter ${ }^{1}$
}

\section{Effects of the home learning environment on children's emerging literacy}

\section{Zusammenfassung:}

Der Erwerb von Lesen, als eine der zentralen Kulturtechniken unserer Gesellschaft, wird bereits früh angebahnt und durch die sozialen Interaktionen des Kindes mit seiner Umwelt gefördert. Als bedeutsamste Umwelt kann wohl die Familie angesehen werden, welche als erste Sozialisationsinstanz die Kinder entscheidend prägt. Doch welche Praktiken genau für die verschiedenen Vorläufer des Lesens von Bedeutung sind, ist bislang, vor allem im deutschsprachigen Raum, recht wenig erforscht. Vor diesem Hintergrund wird im vorliegenden Beitrag die Bedeutsamkeit unterschiedlicher Facetten der familiären Anregung bei 554 Kindern der Längsschnittstudie BiKS-3-10 im Alter von ca. 3-4 Jahren für verschiedene Kompetenzen, die als Vorläufer des Lesens gelten können, untersucht. Es werden drei Dimensionen der familiären Lernumwelt unterschieden: Qualität der Interaktion, formelle Instruktion in Schriftsprache und Erfahrungen mit Büchern. Mit Hilfe eines Pfadmodells wird der Zusammenhang zwischen diesen verschiedenen Dimensionen der fa-

\begin{abstract}
:
The acquisition of reading competencies as a significant cultural skill in our society is initiated early on and supported by a child's interaction with its social surroundings. The family, as probably the most important agent of socialization, decisively affects children and their competencies. However, which forms of activities are meaningful for the development of precursors to reading, known as emergent literacy, has rarely been investigated in Germany yet. Therefore, the present paper explores the importance of different facets of the home learning environment on children's emergent literacy, using data of 554 children, age 3 to 4 years, from the longitudinal study BiKS-3-10. Three dimensions of the home learning environment are distinguished: quality of interaction, formal instruction in literacy, and exposure to books. Through path modeling the associations between these dimensions of the home learning environment were analyzed, controlling for children's level of competencies at the beginning of the study and family background variables
\end{abstract}

1 Die vorliegende Arbeit ist im Rahmen der, von der Deutschen Forschungsgemeinschaft geförderten, interdisziplinären Forschergruppe „Bildungsprozesse, Kompetenzentwicklung und Selektionsentscheidungen im Vorschul- und Schulalter (BiKS)“ (FOR 543) entstanden. Die Konzeptualisierung und Anleitung der Erhebungen erfolgte insbesondere im Rahmen des entwicklungspsychologischen Teilprojekts (Leitung: S. Weinert) sowie des elementarpädagogischen Teilprojekts (Leitung: H.-G. Roßbach). Wir danken der Deutschen Forschungsgemeinschaft für die Förderung der Forschergruppe und den beteiligten Kindern, Eltern, Erziehern und Lehrkräften sowie allen beteiligten Hilfskräften und Mitarbeitern für Ihre engagierte Mitarbeit. 
miliären Lernumwelt, unter Kontrolle des Ausgangsniveaus kindlicher Kompetenzen und familiärer Hintergrundvariablen (sozioökonomischer Status und sprachlicher Hintergrund), mit schriftsprachlichen Vorläuferfähigkeiten (Wortschatz, Grammatik, inhaltliches Wissen und Buchstabenkenntnis) analysiert. Die Ergebnisse zeigen, dass die verschiedenen Dimensionen der familiären Anregung mit unterschiedlichen Vorläufern schriftsprachlicher Fähigkeiten assoziiert sind. Die Befunde werden vor dem Hintergrund theoretischer und praktischer Implikationen diskutiert.

Schlagwörter: Familiäre Lernumwelt, Emergent Literacy, Lesen, Sprache, Kindergartenalter, Längsschnittstudie (socioeconomic status and parental native language), and emergent literacy (vocabulary, grammar, content knowledge, knowledge of letters). Results show that specific dimensions of the home learning environment are associated with specific facets of emergent literacy. The findings are discussed with regard to theoretical and practical implications.

Key words: home learning environment, emergent literacy, reading, language, preschool age, longitudinal study, early childhood education

\section{Theoretischer Hintergrund}

Der Erwerb von Lesekompetenz ist eine zentrale Voraussetzung der Teilhabe am gesellschaftlichen und kulturellen Leben. Gerade bei deutschen Schülern und Schülerinnen ist diese jedoch stärker als in vielen anderen europäischen Ländern an die soziale Herkunft gekoppelt (Baumert/Schümer 2001). Wird das Bedingungsgefüge der Lesekompetenzentwicklung dabei genauer untersucht, so richtet sich der Blick auch auf die frühe Kindheit (vgl. Roßbach/Weinert 2008). Im Zusammenhang mit der Lesekompetenzentwicklung sind sogenannte Vorläufer des Lesens, die im englischen Sprachraum mit Emergent Literacy betitelt werden, von Bedeutung. Neben Aspekten der phonologischen Informationsverarbeitung gelten auch sprachliche Kompetenzen als relevant für späteres Lesen (vgl. z.B. Dickinson/Porche 2011; NICHD ECCRN 2005; Dickinson et al. 2003). Dabei zeigen Ergebnisse der Studie Bildungsprozesse, Kompetenzentwicklung und Selektionsentscheidungen im Vorschul- und Schulalter (BiKS), dass bereits in frühen sprachlichen Kompetenzen im Alter von nur drei Jahren deutliche interindividuelle Unterschiede in Abhängigkeit von der sozialen Herkunft bestehen (Weinert et al. 2010). Für den Einfluss solcher als Strukturmerkmale bezeichneten Faktoren wird unter anderem die familiäre Lernumwelt als wichtiger Mediator diskutiert (vgl. Bradley et al. 2001; Brooks-Gunn/Duncan 1997). Zahlreiche internationale und einige nationale Befunde belegen die Bedeutsamkeit der familiären Lernumwelt für die kindliche Kompetenzentwicklung im Vorschulalter (z.B. Anders et al. 2012; Weinert et al. 2012; Ebert et al. im Druck; Niklas 2010; Melhuish et al. 2008; für einen Überblick Totsika/Sylva 2004). Ein großes Problem bei der Sichtung dieser Befunde stellt allerdings die heterogene Definition und Operationalisierung der familiären Lernumwelt dar. Die im Zusammenhang mit der Entwicklung schriftsprachlicher Kompetenzen am häufigsten beachtete elterliche Aktivität ist das gemeinsame Lesen bzw. das Vorlesen von Büchern (z.B. Sénéchal 2006; Dickinson/Tabors 2001; Jordan et al. 2000; Ewers/Brownson 1999). Dabei konnte zwar gezeigt werden, dass die Häufigkeit dieser Interaktionsform einen positiven Effekt sowohl auf schriftsprachliche (z.B. Buchstabenkenntnis) als auch auf mündliche Sprachkompetenzen (z.B. rezeptiver Wortschatz) aufweist, dieser jedoch mit einer mittleren 
Varianzaufklärung von $8 \%$ nur moderat ausfällt (Bus et al. 1995; Scarborough/Dobrich 1994). Diese moderaten Effekte lassen die Frage aufkommen, inwieweit das gemeinsame Lesen und Vorlesen allein einen geeigneten bzw. ausreichenden Indikator zur Erklärung interindividueller Unterschiede im Leseerwerb darstellt. Einige Autoren schlagen daher vor, das Konzept der Home Learning Environment (HLE) auszuweiten (z.B. van Steensel 2006; Burgess et al. 2002).

Ziel der vorliegenden Untersuchung ist es, unterschiedliche Facetten der familiären Lernumwelt zu beschreiben und deren differenzielle Wirkung auf verschiedene Vorläuferfertigkeiten des Lesens aufzudecken. Dazu werden zunächst der Begriff und die Komponenten von Emergent Literacy genauer beleuchtet und deren Bedeutung für das Lesen und den Leseerwerb herausgestellt. Des Weiteren werden Forschungsbefunde zu Auswirkungen der familiären Lernumwelt mit besonderem Fokus auf unterschiedliche Operationalisierungen und deren methodische Erfassung berichtet.

\section{Emergent Literacy}

Whitehurst und Lonigan (1998) definieren Emergent Literacy als Fertigkeiten, Einstellungen und Wissen von Kindern, die Lesen und Schreiben im konventionellen Sinne anbahnen. Hierzu gehören zum Beispiel Kenntnisse über Konventionen der Schriftsprache, Vorläufer des Schreibens, das Kennen von Graphemen sowie deren Zuordnung zu Lauten. Die zentrale Annahme ist, dass Kompetenzen, die für das Lesen und Schreiben notwendig sind, nicht erst mit dem Eintritt in das formalisierte Bildungssystem, sondern in einem kontinuierlichen Prozess bereits lange zuvor angeeignet werden (Miller 2000). Einen Beleg für die Bedeutsamkeit der Emergent Literacy für späteres Lesen liefert eine Metaanalyse über rund 300 Studien des National Early Literacy Panels (2008; siehe auch für eine Zusammenfassung Shanahan/Lonigan 2010). Als relevante Prädiktoren für das konventionelle Lesen werden neben Buchstabenkenntnis, phonologischer Bewusstheit, schnellem Benennen von Buchstaben und Objekten, ersten Schreibversuchen und dem phonologischen Arbeitsgedächtnis auch das Wissen über schriftsprachlich bedeutsame Konventionen und die mündlichen Sprachkompetenzen aufgeführt. Diese sogenannten Vorläufer des Lesens lassen sich am besten als zwei miteinander in Beziehung stehende Faktoren verstehen (Snow 1999, 1991): die Fertigkeiten, die sich konkret auf die geschriebene Sprache beziehen und solche, die die mündliche Sprache betreffen (vgl. auch Caspe 2009). Fertigkeiten, die sich auf die geschriebene Sprache beziehen sind z.B. Buchstabenkenntnis und phonologische Bewusstheit. Von diesen Fertigkeiten wird angenommen, dass sie eher in Beziehung zur späteren Lesefertigkeit, also dem Enkodieren von Schriftzeichen, stehen, während Fertigkeiten, die die mündliche Sprache betreffen, z.B. der expressive und rezeptive Wortschatz, sich eher als bedeutsam für das Leseverständnis erweisen sollten (Snow 1999, 1991).

Verschiedene Längsschnittstudien belegen hierbei direkte und indirekte Zusammenhänge zwischen Kompetenzen, die die Schriftsprache bzw. mündliche Sprache betreffen und der späteren Lesekompetenz (z.B. Ennemoser et al. 2012; Whitehurst/Lonigan 2002). 


\section{Die Bedeutung der Familie als Lernumwelt}

Die zentrale Bedeutung der Familie für den kindlichen Kompetenzerwerb als erste Sozialisationsinstanz kann als unumstritten gelten. Für die Anregung schriftsprachlicher Vorläuferkompetenzen in der Familie besteht ein im englischsprachigen Raum etablierter Begriff der sogenannten Home Literacy Environment (HLE) (zum Teil auch Family Literacy; z.B. Wasik 2004). Das Konzept der HLE kann im Allgemeinen zwar als Begriff für die familiären Ressourcen und Möglichkeiten gelten, die Kindern bezüglich des Schriftsprach- und mündlichen Spracherwerbs zur Verfügung gestellt werden (Burgess et al. 2002), es beinhaltet jedoch keine allgemeingültige Definition und damit auch keine einheitliche Operationalisierung. Während Britto und Brooks-Gunn (2001) drei Dimensionen der HLE (sprachliche Interaktion, Lernen und sozial-emotionales Klima) vorschlagen, beinhaltet die Faktorstruktur der HLE nach Umek und Kollegen (2005) fünf Dimensionen: Stimulation zum Sprachgebrauch; Vorlesen, Bibliotheks- und Puppentheaterbesuche; gemeinsame Aktivitäten und Gespräche; interaktives Lesen; Stimulation im Sinne der Zone der proximalen Entwicklung. Leseman und de Jong (1998) zufolge beinhaltet die HLE wiederum drei Dimensionen: die Möglichkeit zur Schriftspracherfahrung (z.B. Vorlesen), die Qualität der Instruktion und die sozial-emotionale Qualität. Andere Autoren unterscheiden sogar bis zu zehn Dimensionen der HLE (vgl. Gonzales et al. 2011). Einen eher theoriegeleiteten Konzeptualisierungsvorschlag leisten Sénéchal und Le Fevre (2002) mit dem Home Literacy Model. Sie nehmen zwei distinkte Kategorien der HLE an: eine informelle und eine formelle. Die informelle Dimension umfasst die Erfahrungen mit Büchern allgemein sowie das gemeinsame Lesen bzw. Betrachten eines Bilderbuchs. Als informell wird diese Dimension bezeichnet, weil sie sich nicht direkt auf die geschriebene Sprache als solche bezieht, sondern auf den Inhalt der geschriebenen Sprache. Die formelle Dimension hingegen beinhaltet den Fokus auf die Schriftsprache, z.B. das Alphabet zu unterrichten oder den eigenen Namen zu schreiben. Die Autoren konnten in ihrer Studie zeigen, dass beide Dimensionen nicht signifikant miteinander korreliert sind und damit zwei unterschiedliche Bereiche familiärer Anregung abdecken.

Hinsichtlich der informellen Dimension zeigen z.B. die bereits weiter oben zitierten Metaanalysen, dass ca. 8\% der Varianz der sprachlichen Fertigkeiten der Kinder durch die Häufigkeit des Vorlesens erklärt werden (Bus et al. 1995; Scarborough/Dobrich 1994). Die Arbeiten von Sénéchal (Sénéchal 2006; Sénéchal/LeFevre 2002; Sénéchal et al. 1998) belegen zusätzlich differentielle Effekte: Während die informelle Dimension (story book exposure) positiv mit dem Wortschatzerwerb assoziiert ist, erweist sich die formelle Instruktion als bedeutsam für die Buchstabenkenntnis und Dekodierfähigkeit des Kindes. Auch die bereits erwähnte Metaanalyse des National Early Literacy Panels zur Wirksamkeit von Interventionen zur Förderung schriftsprachlicher Kompetenzen unterscheidet unter anderem zwischen den Interventionen, die darauf gerichtet sind, die Fertigkeiten zum Dekodieren des Alphabets zu erlernen und solchen, die das gemeinsame Buchlesen fördern (vgl. Shanahan/Lonigan 2010). Sie zeigen, dass Interventionen mit einem Fokus auf Dekodierung moderate bis hohe Effektstärken für schriftsprachliche Fertigkeiten, wie phonologische Bewusstheit und Buchstabenkenntnis, aufweisen. Interventionen, die das gemeinsame Buchlesen beinhalten, wiesen dagegen moderate Effektgrößen für mündliche Sprachfertigkeiten und Wissen über Geschriebenes auf. Sénéchal und LeFevre (2002) 
nehmen an, dass informelle Erfahrungen mit Büchern die Sprachkompetenzen der Kinder über die Reichhaltigkeit der Sprache im Text oder aber über die Interaktionsformen der Eltern, z.B. den Gebrauch von Fragen (siehe auch Whitehurst et al. 1988), beeinflussen. Auf Letzteres weisen auch die Befunde des National Early Literacy Panels hin, die höhere Effektstärken für Vorleseinterventionen berichten, die eine interaktive Beteiligung des Kindes, z.B. durch Fragen an das Kind, erforderten (Shanahan/Lonigan 2010).

Die Bedeutsamkeit der Qualität der Interaktion, insbesondere während des gemeinsamen Buchlesens zwischen Betreuungsperson und Kind, konnte in einigen Studien belegt werden (Mol et al. 2009; Mol et al. 2008). Die Qualitätsabstufungen gründen dabei auf den Annahmen der soziokulturellen Theorien, welche die soziale Interaktion eines Kindes mit einem erfahreneren Interaktionspartner als zentral erachten (Wygotski 1969). Auch die Arbeiten von Sigel (1982) zum sogenannten Distancing, welches Verhaltensweisen bzw. sprachliche Äußerungen beinhaltet, die das Kind dazu anregen, von der unmittelbaren Umwelt mental zu abstrahieren, betonen die Anregung zur aktiven Beteiligung des Kindes am Interaktionsprozess. Diese Art der sprachlichen Anregung, die zugleich hohe mentale Anforderungen an das Kind stellt, gilt dabei als positiv assoziiert mit der kognitiven Entwicklung der Kinder (vgl. Sigel et al. 1991; Sigel 1986). Einige Interventionsprogramme knüpfen an diese Überlegungen an und unterrichteten Eltern erfolgreich in dem Gebrauch interaktiver Vorlesestrategien. Whitehurst und Kollegen (1988) entwarfen das Programm des Dialogic Reading, um die Qualität des gemeinsamen Lesens über die erhöhte Beteiligung des Kindes in der gemeinsamen Lesesituation, z.B. durch den Gebrauch offener Fragen und die Verminderung des reinen Vorlesens, zu verbessern. Kinder der Interventionsgruppe zeigten daraufhin einen signifikant höheren expressiven Wortschatz. Darüber hinaus zeigen weitere Studien, dass spezifische elterliche Verhaltensweisen während des Vorlesens von Büchern, wie der Gebrauch offener Fragen, das Hinzufügen von Informationen, das Fokussieren auf die Schriftsprache und der Gebrauch abstrakter Sprache, positiv mit der sprachlichen Entwicklung der Kinder assoziiert sind (Leseman/de Jong 1998; Lonigan/Whitehurst 1998; van Kleeck et al. 1997; Haden et al. 1996; Whitehurst et al. 1994a; Whitehurst et al. 1994b).

Zusammenfassend erscheint eine Unterscheidung in eine informelle und eine formelle Dimension der HLE plausibel. Eine Erweiterung um eine Dimension der Qualität der elterlichen Anregung scheint mit Blick auf die empirischen Befunde allerdings sinnvoll. Vor diesem Hintergrund werden in der vorliegenden Untersuchung drei Dimensionen der HLE (Erfahrungen mit Büchern, Qualität der Interaktion und formelle Instruktion von Schriftsprache) in Anlehnung und gleichzeitiger Erweiterung des Modells von Sénéchal und LeFevre (2002) unterschieden. Im Unterschied zu anderen Studien (z.B. de Jong/Leseman 2001; Niklas 2010) werden in der vorliegenden Studie verschiedene Aspekte der frühen Vorläuferfertigkeiten des Lesens in ihrer Beziehung zu unterschiedlichen Aspekten der HLE längsschnittlich untersucht. Konkret wird bezüglich der HLE zwischen der direkten Instruktion von Schriftsprache als formeller Dimension und der Erfahrung mit Büchern als informeller Dimension differenziert. Ergänzend wird, als weitere informelle Variable, die sprachliche Anregung in der Interaktion als Qualität der Interaktion berücksichtigt. Als zentrale Vorläufer des Lesens werden insbesondere mündliche Sprachfähigkeiten (Wortschatz und Grammatik) sowie das inhaltliche Vorwissen und die Buchstabenkenntnis herangezogen. Dabei werden konkret drei Fragestellungen untersucht: 
(1) In welcher Beziehung stehen die von Sénéchal und LeFevre (2002) vorgeschlagenen Dimensionen der HLE in der vorliegenden Stichprobe und unterstützen diese Beziehungen die Annahme eines multidimensionalen Konstrukts?

(2) Können differentielle Effekte der verschiedenen Dimensionen der HLE auf verschiedene Vorläuferfertigkeiten des Lesens identifiziert werden?

(3) Werden Effekte familiärer Hintergrundmerkmale auf die Emergent Literacy Fertigkeiten über Merkmale der HLE vermittelt?

\section{Methode}

Stichprobe und Vorgehen. Die Daten der vorliegenden Studie wurden im Rahmen der Längsschnittstudie BiKS-3-10 (Bildungsprozesse, Kompetenzentwicklung und Selektionsentscheidungen im Vorschul- und Schulalter) erhoben. Die Stichprobe setzt sich aus 554 Kindern aus 97 (60 bayerischen und 37 hessischen) Kindergärten zusammen. Die Auswahl der Kindergartengruppen erfolgte nach einem mehrfach geschichteten Erhebungsdesign (vgl. Kurz et al. 2007), wobei 65.2\% der Kinder aus Bayern und 34.8\% aus Hessen rekrutiert wurden.

In die Studie wurden nur die Kinder einer Kindergartengruppe aufgenommen, deren fristgerechte Einschulung für das Schuljahr 2008 anstand, um eine in dieser Hinsicht möglichst homogene Stichprobe zu gewinnen. Für die folgenden Analysen wurden Daten innerhalb des ersten (Herbst 2005), zweiten (Herbst 2006) und dritten (Frühjahr 2008) Kindergartenjahres verwendet (im Folgenden als MZP (Messzeitpunkt) 1, 2, 3 bezeichnet). Das mittlere Alter zum ersten Messzeitpunkt betrug 44.5 Monate ( $\mathrm{SD}=4.8$ Monate). Insgesamt wuchsen $12.1 \%$ der Kinder mit einem Elternteil nicht muttersprachlich deutscher Herkunft auf und 9.7\% der Kinder mit zwei Elternteilen, die eine andere Muttersprache als Deutsch aufwiesen.

\section{Messinstrumente}

Familiäre Hintergrundmerkmale. Die familiären Hintergrundmerkmale wurden über ein standardisiertes persönliches Elterninterview zum ersten Messzeitpunkt erfasst. Einbezogen wurden Variablen, die sich für die Kompetenzentwicklung der Kinder im Lebenslauf als besonders bedeutsam erwiesen haben, speziell der sozioökonomische Status der Familie sowie der Migrationshintergrund bzw. sprachliche Hintergrund des Kindes (z.B. Dubowy et al. 2008; Ehmke et al. 2004; Baumert/Schümer 2001).

Sozioökonomischer Status (SES). Als Indikator für den SES wurde der höchste Internationale Sozioökonomische Index (ISEI; vgl. Ganzeboom/Treimann 1996) der Familie herangezogen.

Sprachlicher Hintergrund. Der sprachliche Hintergrund wurde durch eine dichotome Variable erfasst. Ein nicht-deutscher sprachlicher Hintergrund wurde dann kodiert, wenn mindestens ein Elternteil nicht muttersprachlich deutscher Herkunft war. 


\section{Vorläufer schriftsprachlicher Kompetenzen}

Wortschatz. Erfasst wurde der rezeptive Wortschatz der Kinder anhand einer nicht publizierten deutschen Forschungsversion des Peabody Picture Vocabulary Test (PPVT, Dunn/ Dunn 1981; Roßbach et al. 2005). In diesem Test wurde den Kindern je Item ein Wort vorgegeben, $\mathrm{zu}$ dem sie das passende Bild aus vier schwarz-weißen Zeichnungen auswählen sollten. Als Indikator für den rezeptiven Wortschatz wurde die Anzahl richtig beantworteter Items ermittelt $(\operatorname{Max}=175)$. In die Analysen wurden die Leistungen der Kinder zu MZP 1 und 2 einbezogen.

Grammatik. Als Maß für die kindliche Verarbeitung grammatischer Strukturen wurde zu MZP 1 der Subtest „Verstehen von Sätzen“ aus dem Sprachentwicklungstest für 3- bis 5jährige Kinder (SETK 3-5, Grimm 2001) eingesetzt. Zu vorgegebenen Sätzen ist bei einem Teil der Items die Auswahl eines passenden Bildes aus jeweils vier Wahlmöglichkeiten gefordert, bei anderen wird das Ausagieren der Sätze verlangt. Da dieses Testverfahren nur in einem begrenzten Altersbereich einsetzbar ist, wurde zum zweiten Messzeitpunkt des Längsschnitts BiKS-3-10 auf eine gekürzte deutsche Version des Test for the Reception of Grammar (TROG, Bishop 1989; deutsche Version TROG-D von Fox 2006) zurückgegriffen, die zum ersten Messzeitpunkt noch nicht vorlag. Hier wird über einen weiten Altersbereich (3 bis 10 Jahre) gezielt das Verständnis zunehmend komplexer grammatischer Strukturen überprüft. Das Aufgabenformat des TROG-D entspricht jenem, das auch im Untertest „Verstehen von Sätzen“ aus dem SETK 3-5 zum Einsatz kommt (Bildauswahl zu vorgegebenen Sätzen mit variierender grammatischer Struktur). Die gekürzte Version des TROG-D besteht insgesamt aus 48 Items, die in Sets gleicher grammatischer Strukturen zusammengefasst sind. Als Indikator wurde der Summenwert über alle richtig beantworteten Items herangezogen.

Inhaltliches Wissen. Das frühe inhaltliche Wissen der Kinder wurde mit Hilfe des Subtests "Gesichter und Orte“ aus der Kaufman Assessment Battery for Children (K-ABC, deutsche Version: Melchers/Preuß 2003) gemessen. Dieser Subtest erfasst, inwieweit die Kinder verschiedene fiktionale oder reale bekannte Persönlichkeiten (z.B. „Weihnachtsmann“) und Orte (z.B. „Eifelturm“) kennen, die zum allgemeinen Wissensschatz von Kindern der entsprechenden Altersstufe zählen (Melchers/Preuß 2003). Insgesamt wurden den Kindern 16 Bilder gezeigt und sie sollten angeben, wer oder was auf dem Bild abgebildet ist. Der Subtest „Gesichter und Orte“ wurde zu MZP 1 und 2 einbezogen.

Buchstabenkenntnis. Zur Erfassung der Buchstabenkenntnis wurden den Kindern die 26 Buchstaben des Alphabets in 5-er bzw. 6-er Gruppen auf ca. 20 x $15 \mathrm{~cm}$ großen Bildkarten vorgegeben, wobei die Buchstaben eine Höhe von ca. $2 \mathrm{~cm}$ hatten und zufällig auf den für alle Kinder identischen Tafeln angeordnet waren. Bei der Konstruktion der Tafeln wurde darauf geachtet, dass keine Buchstaben aufeinander folgen, die im Alphabet nacheinander stehen. Die Kinder wurden je Tafel aufgefordert, die Buchstaben zu benennen, die sie kennen. Als richtig wurde sowohl die formal korrekte, als auch die lautgetreue Aussprache gewertet. In den Analysen wurde die Anzahl richtig identifizierter Buchstaben berücksichtigt, wobei in der vorliegenden Studie die Buchstabenkenntnis der Kinder zum dritten Messzeitpunkt eingeht. 
Die „Home literacy environment (HLE)“. Die Anregung in der Familie wurde zum einen über Befragungsvariablen einer adaptierte Version der Home Observation for Measurement of the Environment (HOME, Caldwell/Bradley 1984) und zum anderen über ein innerhalb der Forschergruppe entwickeltes Live-Rating (Familieneinschätzskala (FES)), einer halbstandardisierten Bilderbuchsituation zwischen der Hauptbetreuungsperson und dem Kind zu MZP 1, erfasst (Kuger et al. 2005). Auf Grundlage dieser Daten wurden für die vorliegende Untersuchung folgende Skalen zur häuslichen Anregung im Bereich (Schrift-)Sprache, über die Berechnung von Mittelwerten der entsprechenden Items, gebildet:

Formelle Instruktion von Schriftsprache. Diese Skala wurde in Anlehnung an die Arbeiten von Sénéchal analog zur Dimension parental teaching of literacy konstruiert und beinhaltet zwei Items des Elternfragebogens, die auf einer vierstufigen Skala $(1=$ selten oder nie, 2 = manchmal, 3 = regelmäßig, 4 = häufig) eingeschätzt werden sollten. Beide Items entstammen der HOME (Caldwell/Bradley 1984): „Anhand einfacher Wörter ermutige ich mein Kind, Buchstaben und Wörter zu erkennen oder zu lesen, z.B. den eigenen Namen oder den Namen seines Lieblingstieres“; „Mein Kind soll sich mit dem Alphabet vertraut machen, z.B. durch das Schreiben seines eigenen Namens, Besprechen einzelner Buchstaben in Büchern, Zeitungen oder an einer Tafel“". Die Items korrelieren mit $\mathrm{r}=.77$.

Erfahrung mit Büchern. In Anlehnung an die Messung der story book exposure von Sénéchal und LeFevre (2002) beinhaltet diese Skala die Angaben der Eltern zur Anzahl der Kinder- und Erwachsenenbücher sowie zur Häufigkeit des Vorlesens. Die Angaben der Eltern wurden aufgrund unterschiedlicher Antwortskalen zunächst standardisiert und anschließend gemittelt (Cronbachs Alpha $=.68)$.

Qualität der Eltern-Kind-Interaktion. Diese Skala wurde auf Grundlage des Live-Ratings mithilfe der FES gebildet und beinhaltet sechs Items die sich auf den Gebrauch von Fragen, die verbale Distanzierung, Gesprächsanteile, das nonverbale Verhalten, das Niveau der freien Sprache und das Geben phonologischer Hinweise beziehen. Die Einschätzung erfolgte auf einer Skala von 1 ( = unzureichend) bis 7 ( = ausgezeichnet). Die interne Konsistenz (Cronbachs Alpha) ist mit .65 zufriedenstellend.

\section{Statistische Analyse}

Zur Beantwortung der Fragestellungen wurde ein Pfadmodell spezifiziert, welches $\mathrm{Ab}$ bildung $1 \mathrm{zu}$ entnehmen ist. Alle Analysen erfolgten mit den Statistikprogrammen SPSS 19.0 und Mplus 6.0 (Muthén/Muthén 1998-2011). Um eine durch das längsschnittliche Design bedingte Reduktion der Stichprobengröße und damit eventuell einhergehende verzerrte Schätzungen zu vermeiden, wurde für die vorliegenden Analysen die Full Information Maximum Likelihood (FIML) gewählt, welche in Mplus implementiert ist. Diese ermöglicht die Modellschätzung unter Berücksichtigung aller Fälle auch bei einzelnen fehlenden Werten (Arbuckle 1996). 


\section{Ergebnisse}

Zur Untersuchung der Beziehung zwischen den familiären Anregungsmerkmalen untereinander und deren Bedeutung für die frühen schriftsprachrelevanten Kompetenzen der Kinder (emergent literacy) wurde ein Pfadmodell spezifiziert. Hierzu wurden Regressionspfade von den drei Skalen der familiären Anregungsmerkmale zum ersten Messzeitpunkt zu den Kompetenzvariablen zum zweiten bzw. dritten Messzeitpunkt modelliert. Zur Interpretation der Ergebnisse im Sinne eines Zuwachses wurden die Kompetenzen zum ersten Messzeitpunkt über autoregressive Pfade zu den Kompetenzen zum zweiten bzw. dritten Messzeitpunkt spezifiziert. Zugleich wurden familiäre Hintergrundvariablen (sprachlicher und sozioökonomischer Hintergrund) berücksichtigt, indem jeweils Pfade hin zu den verschiedenen Kompetenzvariablen sowie den familiären Anregungsmaßen angenommen wurden.

Darüber hinaus wurden die messzeitpunktgleichen Korrelationen zwischen den Anregungsmerkmalen und den Kompetenzen berücksichtigt.

Das so spezifizierte Modell ist Abbildung $1 \mathrm{zu}$ entnehmen. Aus Gründen der Übersichtlichkeit wurden jedoch lediglich die signifikanten Regressionspfade der familiären Hintergrund- und Anregungsmerkmale auf die Kompetenzen zum zweiten bzw. dritten Messzeipunkt eingezeichnet. Aus demselben Grund wurde auch auf die Einzeichnung der Korrelationen verzichtet. Diese sind oberhalb der Diagonalen der Tabelle $1 \mathrm{zu}$ entnehmen.

Bei dem vorliegenden Pfadmodell handelt es sich um ein saturiertes Modell.

Abbildung 1: Modell zur Bedeutung der familiären Lernumwelt für Vorläufer schriftsprachlicher Kompetenzen.

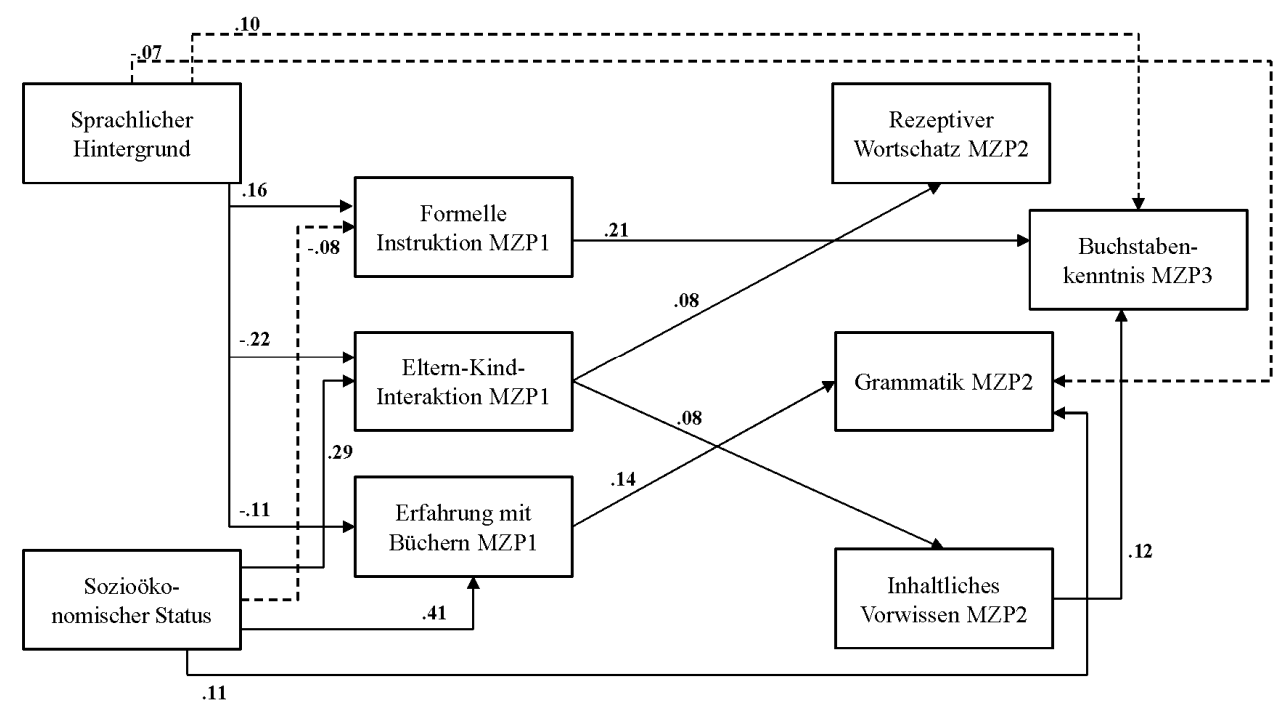

Anmerkungen: Darstellung der statistisch signifikanten, standardisierten Pfadkoeffizienten $\mathrm{p}<.05$; gestrichelte Pfade statistisch signifikant auf dem 10\%-Niveau; Sprachlicher Hintergrund: $0=$ beide Elternteile muttersprachlich deutsch, 1 = mindestens ein Elternteil nicht-deutsche Muttersprache; Kompetenzen zu MZP 2 und 3 kontrolliert für Kompetenzen zu MZP 1 (rezeptiver Wortschatz, Grammatik, inhaltliches Vorwissen); Korrelationen siehe Tabelle 1. 
Tabelle 1: Im Rahmen des Pfadmodells spezifizierte Korrelationen (oberhalb der Diagonale) sowie bivariate Korrelationen zwischen den Kompetenz-, Hintergrundund HLE-Variablen (unterhalb der Diagonale)

\begin{tabular}{|c|c|c|c|c|c|c|c|c|c|c|c|c|}
\hline & 1. & 2. & 3. & 4. & 5. & 6. & 7. & 8. & 9 & 10 & 11 & 12 \\
\hline 1. Wortschatz (MZP 1) & - & $.64^{\star *}$ & $.54^{* *}$ & & & & & & & $.20^{\star *}$ & $.11^{* *}$ & $.19^{* *}$ \\
\hline 2. Grammatik (MZP 1) & $.71^{* *}$ & - & $.45^{\star *}$ & & & & & & & $.12^{*}$ & $.14^{* *}$ & $.13^{* *}$ \\
\hline 3. Inhaltliches Wissen (MZP 1) & $.59^{\star *}$ & $.52^{\star *}$ & - & & & & & & & $.20^{\star *}$ & $.13^{* *}$ & .08 \\
\hline 4. Wortschatz (MZP 2) & $.68^{* *}$ & $.67^{* *}$ & $.52^{* *}$ & - & $.25^{\star *}$ & $.19^{* *}$ & & & & & & \\
\hline 5. Grammatik (MZP 2) & $.61^{* *}$ & $.63^{\star \star}$ & $.42^{* \star}$ & $.62^{* *}$ & - & $.10^{*}$ & & & & & & \\
\hline 6. Inhaltliches Wissen (MZP 2) & $.57^{* *}$ & $.51^{* *}$ & $.71^{* *}$ & $.56^{\star *}$ & $.46^{\star *}$ & - & & & & & & \\
\hline 7. Buchstaben (MZP 3) & $.24^{\star *}$ & $.24^{* *}$ & $.24^{* *}$ & $.24^{\star *}$ & $.20^{* *}$ & $.27^{* *}$ & - & & & & & \\
\hline 8. sprachlicher Hintergrund & $-.28^{* *}$ & $-.36^{\star \star}$ & $-.16^{\star *}$ & $-.30^{\star \star}$ & $-.29^{\star \star}$ & $-.19^{* *}$ & -.01 & - & & & & \\
\hline 9. SES (Hisei) & $.32^{* *}$ & $.41^{* *}$ & $.24^{* *}$ & $.31^{* *}$ & $.40^{* *}$ & $.28^{* *}$ & $.17^{* *}$ & $-.21^{* *}$ & - & & & \\
\hline 10. Formelle Instruktion & $.12^{*}$ & .01 & $.15^{\star *}$ & .06 & .02 & .08 & $.23^{* *}$ & $.15^{\star *}$ & $-.12^{*}$ & - & .06 & .06 \\
\hline 11. Qualität der Interaktion & $.27^{\star \star}$ & $.33^{* *}$ & $.24^{\star *}$ & $.32^{* \star}$ & $.22^{\star *}$ & $.30^{* *}$ & $.11^{*}$ & $-.26^{\star *}$ & $.34^{* *}$ & -.00 & - & $.13^{* *}$ \\
\hline 12. Erfahrungen mit Büchern & $.28^{* *}$ & $.28^{* *}$ & $.15^{* *}$ & $.25^{\star *}$ & $.36^{\star *}$ & $.20^{* *}$ & $.16^{* *}$ & $-.16^{\star *}$ & $.42^{* *}$ & -.01 & $.27^{* *}$ & \\
\hline
\end{tabular}

Anmerkungen:

Sprachlicher Hintergrund: $0=$ beide Elternteile muttersprachlich Deutsch, $1=$ mindestens ein Elternteil nicht-deutsche Muttersprache. SES = sozioökonomischer Status

$* \mathrm{p}<.05 . * * \mathrm{p}<.01$.

Zusammenhänge zwischen den Dimensionen der HLE. Um entsprechend der ersten Forschungsfrage zu prüfen, welche Beziehung zwischen den drei Dimensionen der HLE besteht, werden die Korrelationen, die im Rahmen des Gesamtmodells geschätzt wurden, zwischen der formellen Dimension „Formelle Instruktion von Schriftsprache“ und den beiden informellen Dimensionen „Erfahrung mit Büchern“ und „Qualität der Eltern-KindInteraktion" herangezogen. Tabelle 1 ist zu entnehmen, dass die beiden informellen Dimensionen Erfahrungen mit Büchern und Qualität der Interaktion positiv miteinander in Beziehung stehen $(\mathrm{r}=.13, \mathrm{p}<.01)$, während die formelle Instruktion der Schriftsprache mit keiner der beiden Dimensionen in signifikantem Zusammenhang steht (jeweils $r=$ $.06, \mathrm{~ns}$ ). Auch die einfachen bivariaten Korrelationen stützen diesen Befund (vgl. Tabelle 1; unterhalb der Diagonale). Eltern, die ihr Kind vergleichsweise häufig direkt in der Schriftsprache unterweisen, ermöglichen ihrem Kind nicht zugleich vergleichsweise häufigere Erfahrungen mit Büchern oder qualitativ anregende Interaktionen. Zudem weist dieser Befund darauf hin, dass eine Differenzierung in eine formelle und eine informelle Dimension der HLE sinnvoll ist. Auch die nur moderate Beziehung zwischen den beiden Facetten der informellen Dimension stützt die Vorgehensweise, zwischen der „Erfahrung mit Büchern“ und der „Qualität der Eltern-Kind-Interaktion“ zu unterscheiden.

Bedeutung der Dimensionen der HLE für Vorläufer schriftsprachlicher Kompetenzen. Zur Beantwortung der zweiten Fragestellung, welche die differenzielle Beziehung zwischen Merkmalen der HLE und den schriftsprachlichen Vorläuferfähigkeiten untersucht, werden die in Abbildung 1 dargestellten Regressionspfade herangezogen, die von den Dimensionen der HLE zu den Vorläuferkompetenzen zu MZP 2 und 3 führen. Es finden sich bezüglich der Effekte der familiären Anregungsmerkmale auf die kindlichen Kompetenzmaße unter Kontrolle der familiären Hintergrundmerkmale und Ausgangskompetenzen der Kinder differenzielle Zusammenhänge: Die informelle Dimension der familiären An- 
regung im Sinne der Qualität der Eltern-Kind-Interaktion ist positiv mit dem Wortschatz $(\beta=.08, p<.05)$ sowie dem inhaltlichen Vorwissen der Kinder $(\beta=.08, p<.05)$ assoziiert, jedoch nicht mit dem Grammatikverständnis und der Buchstabenkenntnis. Die zweite informelle Dimension, die Häufigkeit der Erfahrung mit Büchern, erweist sich lediglich für den Grammatikerwerb $(\beta=.14, p<.05)$ als bedeutsam. Die Häufigkeit der formellen Instruktion in Schriftsprache hingegen sagt die Buchstabenkenntnis vorher $(\beta=.21, \mathrm{p}<$ $.05)$, steht jedoch mit keinem anderen Kompetenzmaß in Beziehung.

Durch das Modell konnten 56 Prozent der Varianz des Wortschatzes, 55 Prozent der Varianz des Grammatikverständnisses, 50 Prozent der Varianz des Vorwissens und 20 Prozent der Varianz in der Buchstabenkenntnis erklärt werden. Dabei ist zu beachten, dass im Modell kein äquivalentes Maß zu MZP 1 für Buchstabenkenntnis enthalten ist, während sich die höhere Varianzaufklärung für Wortschatz, Grammatik und Vorwissen vor allen Dingen durch die Berücksichtigung entsprechender Kompetenzen zu MZP 1 erklären lässt.

Die Befunde deuten darauf hin, dass unter Kontrolle der frühen kindlichen Emergent Literacy (Wortschatz, Grammatik, Vorwissen) zum ersten Messzeitpunkt sowie unter Kontrolle familiärer Hintergrundmerkmale, Kinder in Familien mit qualitativ anregenderen Interaktionen einen größeren Zuwachs im rezeptiven Wortschatz und im inhaltlichen Vorwissen aufweisen. Kinder hingegen, die häufiger Erfahrungen mit Büchern machen, zeigen einen größeren Zuwachs im Grammatikverständnis. Die direkte Unterweisung in Schriftsprache geht schließlich mit einer besseren Buchstabenkenntnis einher.

HLE als Mediator sozialer Hintergrundvariablen. Um gemäß der dritten Fragestellung zu beantworten, ob Effekte des familiären Hintergrunds auf die Emergent Literacy der Kinder über die HLE vermittelt werden, wurde anhand des Pfadmodells geprüft, inwieweit signifikante indirekte Pfade von den familiären Hintergrundvariablen über die HLE auf die kindlichen Fähigkeiten vorliegen. Dabei ist zu berücksichtigen, dass der sprachliche Hintergrund als auch der sozioökonomische Hintergrund signifikante Zusammenhänge ohne Berücksichtigung der HLE mit allen hier gemessenen kindlichen Fähigkeiten aufweisen (vgl. Tabelle 1).

Wie Abbildung $1 \mathrm{zu}$ entnehmen ist, wurden indirekte Pfade zwischen dem sozioökonomischen Status bzw. dem sprachlichen Hintergrund über die HLE auf die Kompetenzen zum zweiten bzw. dritten Messzeitpunkt spezifiziert. Diese Spezifizierung ermöglicht die Testung indirekter Effekte, welche sowohl durch die konventionelle Signifikanztestung als auch durch die Berechnung bias-korrigierter Bootstrapping-Konfidenzintervalle abgesichert wurden (MacKinnon 2008; MacKinnon et al. 2004).

Die Analysen zeigen bedeutsame indirekte Effekte des sozioökonomischen Hintergrundes und des sprachlichen Hintergrundes auf die Kompetenzen zum 2. bzw. 3. Messzeitpunkt, vermittelt über die familiären Anregungsmerkmale. Dabei konnten, je nach Kompetenzmaß, unterschiedliche indirekte Effekte festgestellt werden: Die informelle Dimension „Qualität der Interaktion“ vermittelt den Effekt des SES und des sprachlichen Hintergrundes auf den Wortschatz $(\beta=.02, p<.05$ bzw. $\beta=-.02, p<.05)$ und das inhaltliche Wissen $(\beta=.02, p<.05$ bzw. $\beta=-.02, p<.05)$. Beim Grammatikverständnis hingegen konnten zwar indirekte Effekte des SES und des sprachlichen Hintergrundes über die informelle Dimension „Erfahrung mit Büchern“ festgestellt werden $(\beta=.06, p<.01$ bzw. $(\beta=-.01, p<.10)$, zusätzlich wiesen beide Hintergrundmerkmale auch einen direkten Ef- 
fekt auf $(\beta=.11, p<.01$ bzw. $\beta=-.07, p<.05)$. Für die Buchstabenkenntnis konnten weder indirekte noch direkte Effekte der familiären Hintergrundmerkmale festgestellt werden.

\section{Diskussion}

Ziel der vorliegenden Untersuchung war es zum einen, verschiedene Dimensionen der HLE abzubilden und zum anderen deren Bedeutung für die verschiedenen Vorläufer schriftsprachlicher Kompetenzen im Vorschulalter zu untersuchen. Dazu wurden zunächst drei Dimensionen der HLE in Anlehnung an das Modell von Sénéchal und LeFevre (2002) spezifiziert: Zwei informelle Dimensionen, Erfahrung mit Büchern und Qualität der Interaktion, sowie eine formelle Dimension, die formelle Instruktion von Schriftsprache. Die Distinktheit der drei Kategorien konnte zum einen über geringe bzw. nicht vorhandene korrelative Zusammenhänge der Dimensionen untereinander und deren unterschiedliche Zusammenhänge mit Merkmalen des familiären Hintergrundes sowie zum anderen über deren differentielle Wirkung auf Vorläufer der Schriftsprachkompetenzen gezeigt werden.

Die geringen Korrelationen der Dimensionen untereinander stehen in Einklang mit der Literatur. Auch Sénéchal und LeFevre (2002, siehe auch Sénéchal et al. 1998) sowie Evans und Kollegen (2000) finden keine Zusammenhänge zwischen Erfahrungen mit Büchern und der direkten Unterweisung in der Schriftsprache. Auch bezogen auf die Qualität der Interaktion lassen sich in der vorliegenden Studie ähnliche Zusammenhänge wie z.B. bei Leseman und de Jong (1998) nachweisen. Diese finden eine Korrelation von $\mathrm{r}=.24$ zwischen den Erfahrungen mit Büchern (bei ihnen literacy opportunity) und der Qualität der Interaktion (instruction quality), wobei in der vorliegenden Studie eine numerisch ähnliche Korrelation von $\mathrm{r}=.27 \mathrm{zu}$ beobachten ist. Zwar könnte die Distinktheit der Dimensionen der HLE angezweifelt werden, da diese über unterschiedliche Methoden erfasst wurden, ein Methodeneffekt scheint jedoch weitestgehend ausgeschlossen werden zu können, da die Dimensionen formelle Instruktion und Erfahrungen mit Büchern beide über Befragungen erfasst wurden und nicht in Beziehung zueinander stehen $(\mathrm{r}=.06, \mathrm{p}$ $>$.10). Bei einem Methodeneffekt würde man von einer zumindest minimalen gemeinsamen Fehlervarianz ausgehen.

Für eine notwendige Differenzierung zwischen den Dimensionen der HLE erscheint noch bedeutsamer, dass die verschiedenen Dimensionen auf unterschiedliche Art und Weise mit den Vorläufern schriftsprachlicher Kompetenzen assoziiert sind. Erfahrungen, welche eher informelle Interaktionen mit Geschriebenem beinhalten, wie das gemeinsame Lesen eines Buches und die Qualität der sprachlichen Interaktion während einer gemeinsamen Lesesituation, stehen in Zusammenhang zu sprachlichen Vorläuferkompetenzen wie Wortschatz, Grammatik und Vorwissen. Erfahrungen hingegen, die eine eher formelle Unterweisung in der Schriftsprache beinhalten, gehen mit größerer Buchstabenkenntnis einher, welche die eher schriftsprachbezogenen Kompetenzen der Emergent Literacy widerspiegeln.

Ein weiterer zentraler Befund der vorliegenden Studie ist, dass eine Dimension, die die Häufigkeit des Vorlesens bzw. die Erfahrungen mit Geschriebenem im Allgemeinen 
beinhaltet, zusätzlich einer Erweiterung um Qualitätsaspekte aus einer direkten Interaktionssituation bedarf. Während die bivariaten Korrelationen allein noch auf einen Zusammenhang zwischen den Erfahrungen mit Büchern und den Emergent Literacy Fertigkeiten hinweisen, finden sich keine signifikanten Effekte für den rezeptiven Wortschatz und das allgemeine Vorwissen, wenn zugleich die Qualität der Interaktionssituation berücksichtigt wird. Auch andere Studien, die die Qualität der Interaktion nicht berücksichtigen, zeigen Effekte der Häufigkeit des Vorlesens (z.B. Sénéchal/LeFevre 2002; Cunningham/Stanovich 1991). Die Ergebnisse der vorliegenden Studie weisen darauf hin, dass die Bedeutung des Vorlesens bzw. die Erfahrungen, die Kinder mit Büchern und Geschriebenem machen, für die Entwicklung schriftsprachlicher Kompetenzen weniger auf das Vorlesen und die Erfahrungen mit Büchern selbst zurückzuführen ist, sondern auf die Art und Weise, wie in Vorlesesituationen zwischen Kind und Eltern interagiert wird. Weitere Analysen werden genauer zeigen müssen, welche Rolle die Erfahrungen mit Büchern im Zusammenspiel mit der Qualität der Interaktion und der Häufigkeit der formellen Instruktion bei der Vorhersage kindlicher Kompetenzen spielen. Einschränkend ist festzuhalten, dass dies nur mit Blick auf den Wortschatz und das inhaltliche Wissen gilt, während für den Zuwachs in der Grammatik ein Effekt der Erfahrungen mit Büchern bestehen bleibt, auch wenn zugleich die Qualität der Interaktion berücksichtigt wird. Die Bedeutung der Erfahrung mit Büchern könnte darauf zurück geführt werden, dass Kinder durch Vorlesen linguistisch komplexere Sätze hören (Mason/Allen 1986), da die durchschnittliche Länge von Äußerungen beim Vorlesen größer ist als bei anderen Aktivitäten (Crain-Thoreson et al. 2001) und der elterliche Buchbesitz bzw. die elterliche Leseaktivität auch mit der Verwendung komplexerer mündlicher Sprache einhergeht (Stanovich/ West 1989). Ob es die Erfahrungen mit Büchern oder diese Merkmale des Sprachangebotes sind, die den Grammatikerwerb beeinflussen, kann auf Basis der vorliegenden Studie nicht beurteilt werden. Gerade bei Kindern mit Migrationshintergrund ist es möglicherweise wichtig, dass sie für den Grammatikerwerb in der Mehrheitssprache ausreichend Erfahrungen mit schriftsprachlichem Material der Mehrheitssprache des Landes, in dem sie leben, haben. Dies könnte vor allem dann eine Rolle spielen, wenn die Eltern aufgrund einer anderen Muttersprache nur über eingeschränkte Kompetenzen verfügen. Die von uns erfasste Qualität der Interaktion scheint dagegen stärker für inhaltliches Wissen und weniger für die Grammatik von Bedeutung zu sein. Eine Begründung hierfür könnte sein, dass das verwendete Ratingsystem (FES) möglicherweise keine Aspekte der Eltern-Kind-Interaktion misst, die für den Grammatikerwerb förderlich sind. Dafür spricht auch, dass bei der Vorhersage der Grammatik, im Unterschied zu Wortschatz und Vorwissen, auch direkte Effekte des sozioökonomischen Status und des Migrationshintergrunds bestehen bleiben, die vermutlich zusätzlich noch konfundiert sind (vgl. Dubowy et al. 2008). Zukünftige Forschung wird daher zeigen müssen, über welche Mechanismen das gemeinsame Buchlesen den Grammatikerwerb beeinflusst.

Zusammenfassend ist festzuhalten, dass die vorliegende Studie die bereits gewonnenen Erkenntnisse aus zahlreichen Studien, die die Bedeutung der familiären Lernumwelt für die kindliche Kompetenzentwicklung, meist unter Berücksichtigung einer einzelnen Skala belegen (z.B. Ebert et al. im Druck; Griffin/Morrison 1997; Melhuish et al. 2008; Niklas 2010; Weinert et al. 2012), substantiell erweitern konnte. Zum ersten Mal konnte an einer deutschen Stichprobe gezeigt werden, dass verschiedene Dimensionen der HLE diffe- 
renziert werden können, die sich konzeptionell und in ihrer methodischen Herangehensweise unterscheiden.

In theoretischer Hinsicht sind diese Befunde mit dem Zweikomponenten-Modell der Leseentwicklung von Snow $(1999,1991)$ sowie den empirischen Befunden von de Jong und Leseman (2001) sowie Sénéchal und LeFevre (2002) vereinbar. In diesem Modell werden solche Fertigkeiten, die für das Dekodieren der Schriftzeichen und den frühen Leseerwerb notwendig sind, von den Fertigkeiten unterschieden, die sich auf das spätere Leseverständnis beziehen. Im Bereich der Vorläuferfertigkeiten für Lesen wird in diesem Zusammenhang von inside-out- und outside-in-Fertigkeiten und -Prozessen gesprochen (Whitehurst/Lonigan 1998) bzw. von code-related und language-related skills (Storch/ Whitehurst 2002). Wird also davon ausgegangen, dass die spätere Lesekompetenz durch diese beiden Komponenten bestimmt wird, haben Eltern mehrere Möglichkeiten ihre Kinder zu unterstützen: Zum einen, indem sie über eher informelle Anregungen in der Interaktion, z.B. über das Stellen offener Fragen, das Bereitstellen von Erfahrungen mit Büchern und möglicherweise ein anspruchsvolles Sprachangebot, die sprachlichen Kompetenzen der Kinder fördern und zum anderen, indem sie durch eher formelle Unterweisung die Buchstabenkenntnis und damit die Dekodierfähigkeit unterstützen.

Trotz der Stärken, die die vorliegende Studie aufweist (z.B. die längsschnittliche Betrachtung und der multimethodale Zugang zur HLE) müssen einige Einschränkungen vorgenommen werden. Eine Einschränkung stellt die fehlende Berücksichtigung der quantitativen und qualitativen Erfahrungen der Kinder im Kindergarten dar, die eventuell in Beziehung mit der häuslichen Anregung stehen könnten. Ein positiver Zusammenhang würde bedeuten, dass die Effekte der HLE auch Effekte des Kindergartens widerspiegeln können. Analysen der Forschergruppe zeigen jedoch, dass der quantitative Aspekt, gemessen über die Dauer des Kindergartenbesuches, nicht mit der Qualität der familiären Lernumwelt assoziiert ist (Kluczniok et al. in Druck). Auf der Qualitätsebene zeigen Befunde von Anders und Kollegen (2012) zwar Interaktionseffekte der Kindergartenqualität mit der Qualität der familiären Lernumwelt in der Weise, dass besonders Kinder aus qualitativ anregenden familiären Lernumwelten von einer guten Kindergartenqualität hinsichtlich früher arithmetischer Kompetenzen profitieren, die Haupteffekte für HLE und Kindergartenqualität bleiben jedoch bestehen. Die Modellierung beider Umwelten in einem Modell scheint daher sehr gewinnbringend für die Vorhersage kindlicher Kompetenzen zu sein.

Die vorliegende Studie unterstreicht die Bedeutsamkeit der sprachlichen und schriftsprachlichen Anregung durch die Familie und zeigt, dass Eltern schon früh über verschiedene Interaktionsformen ihren Kindern einen guten Start für die spätere Aneignung des konventionellen Lesens geben können.

\section{Literatur}

Anders, Y., Rossbach, H.-G., Weinert, S., Ebert, S., Kuger, S., Lehrl, S. \& von Maurice, J. (2012). Home and preschool learning environments and their relations to the development of early numeracy skills. Early Childhood Research Quarterly, 27, 2, S. 231-244.

Arbuckle, J. L. (1996). Full information estimation in the presence of incomplete data. In: Marcoulides, G. A. \& Schumacker, R. E: (Hrsg.), Advanced structural equation modeling: Issues and techniques. Mahwah, NJ: Lawrence Erlbaum Associates, S. 243-277. 
Baumert, J. \& Schümer, G. (2001). Familiäre Lebensverhältnisse, Bildungsbeteiligung und Kompetenzerwerb. In: Baumert, J., Klieme, E., Neubrand, M., Prenzel, M., Schiefele, W., Schneider, P., Stanat, P., Tilmann, K.-J. \& Weiß, M.. (Hrsg.), PISA 2000. Basiskompetenzen von Schülerinnen und Schülern im internationalen Vergleich. Opladen: Leske + Budrich, S. 323-407.

Bishop, D. (1989). Test for the Reception of Grammar. Second edition. Manchester: University of Manchester. Manchester, M13 9PL.

Bradley, R. H., Corwyn, R. F., McAdoo, H .P. \& Garcia Coll, C. (2001). The home environments of children in the United States. Part 1: Variations by age, ethnicity, and poverty status. Child Development, 72, 6, S. 1844-1867.

Britto, P. R. \& Brooks-Gunn, J. (Eds.). (2001). The role of family literacy environments in promoting young children's emerging literacy skills. San Francisco: Jossey-Bass (New Directions for Child and Adolescent Development 92),

Brooks-Gunn, J. \& Duncan, G. J. (1997). The effects of poverty on children. Future of Children, 7, 2, S: 55-71.

Burgess, S., Hecht, S. \& Lonigan, C. (2002). Relations of the home literacy environment to the development of reading related abilities: A one-year longitudinal study. Reading Research Quarterly, 37, 4 , S. 408-426.

Bus, A. G., van IJzendoorn, M. H. \& Pellegrini, A. D. (1995). Joint book reading makes for success in learning to read: A meta-analysis on intergenerational transmission of literacy. Review of Educational Research, 65, 1, S. 1-21.

Caldwell, B. \& Bradley, R. (1984). Home observation for measurement of the environment. Little Rock: University of Arkansas at Little Rock.

Caspe, M. (2009). Low-income Latino mothers' booksharing styles and children's emergent literacy development. Early Childhood Research Quarterly, 24, 3, S. 306-324.

Crain-Thoreson, C., Dahlin, M.P. \& Powell, T.A. (2001). Parent-child conversation in three conversational contexts: Variations in style and strategy. In: Rebello Britto, P. \& Brooks-Gunn, J. (Hrsg.), The role of family literacy environments in promoting young children's emergent literacy skills. San Francisco: Jossey-Bass, S. 23-38.

Cunningham, A. E. \& Stanovich, K. E. (1991). Tracking the unique effects of print exposure in children: Associations with vocabulary, general knowledge, and spelling. Journal of Educational Psychology, 83, 2, S. 264-274.

De Jong, P. F., \& Leseman, P. P. M. (2001). Lasting effects of home literacy on reading achievement in school. Journal of School Psychology, 39, 5, S. 389-414.

Dickinson, D. K. \& Porche, M. V. (2011). Relation between language experiences in preschool classrooms and children's kindergarten and fourth-grade language and reading abilities. Child Development, 82,3 , S. 870-886.

Dickinson, D. K. \& Tabors, P. O. (2001). Beginning literacy with language. Baltimore: Brookes.

Dickinson, D. K., McCabe, A., Anastasopoulos, L., Peisner-Feinberg, E. \& Poe, M. (2003). The comprehensive language approach to early literacy: The interrelationships among vocabulary, phonological sensitivity, and print knowledge among preschool-aged children. Journal of Educational Psychology, 95, 3, S. 465-481.

Dubowy, M., Ebert, S., von Maurice, J. \& Weinert, S. (2008). Sprachlich-kognitive Kompetenzen beim Eintritt in den Kindergarten. Ein Vergleich von Kindern mit und ohne Migrationshintergrund. Zeitschrift für Entwicklungspsychologie und Pädagogische Psychologie, 40, 3, S. 124-134.

Dunn, L.M. \& Dunn, L. M. (1981). Peabody Picture Vocabulary Test-Revised. Circle Pines, MN: American Guidance Service.

Ebert, S., Lockl, K., Weinert, S., Anders, Y., Kluczniok, K. \& Rossbach, H.-G. (im Druck). Internal and external influences on vocabulary development in preschool age. Special Edition of School Effectiveness School and School Improvement.

Ehmke, T., Hohensee, F., Heidemeier, H. \& Prenzel, M. (2004). Familiäre Lebensverhältnisse, Bildungsbeteiligung und Kompetenzerwerb. In: Prenzel, M., Baumert, J., Blum, W., Lehmann, R., Leutner, D., Neubrand, M. et al. (Hrsg.), Der Bildungsstand der Jugendlichen in Deutschland - Ergebnisse des zweiten internationalen Vergleichs. Münster: Waxmann, S. 225-254. 
Ennemoser, M., Marx, P., Weber, J. \& Schneider, W. (2012). Spezifische Prädiktoren des Rechtschreibens, der Lesegeschwindigkeit und des Leseverständnisses im deutschen Sprachraum. Evidenz aus zwei Längsschnittstudien vom Kindergarten bis zur vierten Klasse. Zeitschrift für Entwicklungspsychologie und pädagogische Psychologie, 44, 2, S. 53-64.

Evans, C. Shaw, D. \& Bell, M. (2000). Home literacy activities and their influence on early literacy skills. Canadian Journal of Experimental Psychology, 54, 2, S. 65-75.

Ewers, C. A. \& Brownson, S. M. (1999). Kindergartners' vocabulary acquisition as a function of active vs. passive storybook reading, prior vocabulary, and working memory. Journal Reading Psychology, 20, 1, S. 11-20.

Fox, A.V. (2006): TROG-D. Test zur Überprüfung des Grammatikverständnisses. Idstein: SchulzKirchner.

Ganzeboom, H. B. G. \& Treiman, D. J. (1996). Internationally comparable measures of occupational status for the 1988 International Standard Classification of Occupations. Social Science Research, 25, 3, S. 201-239.

Gonzalez, J. E., Taylor, A. B., McCormick, A. S., Villarreal, V., Kim, V., Perez, E. \& Darensbourg, A. (2011). Exploring the underlying factor structure of the English version and Spanish translation of the "Familia" family literacy inventory: A cautionary tale. Early Childhood Research Quarterly, 26, 4, S. 475-483.

Griffin, E. A. \& Morrison, F. J. (1997). The unique contribution of home literacy environment to differences in early literacy skills. Early Child Development and Care, 127, 1, S. 233-243.

Grimm, H. (2001). Sprachentwicklungstest für drei- bis fünfjährige Kinder (SET-K 3-5). Göttingen: Hogrefe.

Haden, C. A., Reese, E. \& Fivush, R. (1996). Mothers' extratextual comments during storybook reading: Stylistic differences over time and across texts. Discourse Processes, 21, 2, S. 135-169.

Jordan, G.E., Snow, C.E. \& Porsche, M.V. (2000). Project EASE: The effect of a family literacy project on kindergarten students' early literacy skills. Reading Research Quarterly, 35, 4, S. 524-546.

Kluczniok, K., Lehrl, S., Kuger, S. \& Roßbach, H.-G. (in Druck). Quality of the home learning environment during preschool age - Domains and contextual conditions. European Early Childhood Education Research Journal.

Kuger, S., Pflieger, K. \& Roßbach, H.-G. (2005). Familieneinschätzskala (Forschungsversion). Bamberg: DFG-Forschergruppe BiKS.

Kurz, K., Kratzmann, J. \& von Maurice, J. (2007). Die BiKS-Studie. Methodenbericht zur Stichprobenziehung. http://psydok.sulb.uni-saarland.de/volltexte/2007/990/pdf/Methodenbericht_2007.pdf [Stand: 2012-03-22].

Leseman, P. P. M. \& de Jong, P. F. (1998). Home literacy: Opportunity, instruction, cooperation and social-emotional quality predicting early reading achievement. Reading Research Quarterly, 33, 3, S. 294-319.

Lonigan, C. J. \& Whitehurst, G. J. (1998). Relative efficacy of parent and teacher involvement in a shared-reading intervention for preschool children from low-income backgrounds. Early Childhood Research Quarterly, 13, 2, S. 263-290.

MacKinnon, D. P. (2008). Introduction to statistical mediation analysis. New York: Lawrence Erlbaum Associates.

MacKinnon, D. P., Lockwood, C. \& Williams, J. (2004). Confidence limits for the indirect effect: Distribution of the product and resampling methods. Multivariate Behavioral Research, 39, 1, S: 99-128.

Mason, J. \& Allen, J. B. (1986). A review of emergent literacy with implications for research and practice in reading. Review of Research in Education, 13, S. 3-47.

Melchers, P. \& Preuß, U. (2003). K-ABC: Kaufman-Assessment Battery for Children. Durchführungsund Auswertungshandbuch. Frankfurt am Main: Swets \& Zeitlinger. (Deutschsprachige Fassung, 6. Auflage).

Melhuish, E. C., Phan, M. B., Syla, K., Sammons, P., Siraj-Blatchford, I. \& Taggart, B. (2008). Effects of home learning environment and preschool centre experience upon literacy and numeracy development in early primary school. Journal of Social Issues, 64, 1, S. 95-114. 
Miller, W. (2000). Strategies for developing emergent literacy. Boston: Mc Graw Hill.

Mol, S., Bus, A., de Jong, M., \& Smeets, D. (2008). Added value of dialogic parent-child book readings: A meta-analysis. Early Education and Development, 19, 1, S: 7-26.

Mol, S., Bus, A., \& de Jong, M. (2009). Interactive book reading in early education: A tool to stimulate print knowledge as well as oral language. Review of Educational Research, 79, 2, S. 979-1007.

Muter, V., Hulme, C. \& Snowling, M. J. \& Stevenson, J. (2004). Phonemes, rimes, vocabulary and grammatical skills as foundations of early reading development: Evidence from a longitudinal study. Developmental Psychology, 40, 5, S. 663-681.

Muthén, L. K., \& Muthén, B. O. (1998-2011). Mplus user's guide. Los Angeles, CA: Muthén \& Muthén. $\left(6^{\text {th }}\right.$ edition $)$.

National Early Literacy Panel. (2008). Developing early literacy: Report of the National Early Literacy Panel. Washington, DC: National Institute for Literacy.

NICHD ECCRN (2005). Pathways to reading: The role of oral language in the transition to reading. Developmental Psychology, 41, 2, S. 428-442.

Niklas, F. \& Schneider, W. (2010). Der Zusammenhang von familiärer Lernumwelt mit schulrelevanten Kompetenzen im Vorschulalter. Zeitschrift für Soziologie der Erziehung und Sozialisation, 30, 2, S. 149-165.

Roßbach, H.-G., Tietze, W. \& Weinert, S. (2005). Peabody Picture Vocabulary Test - Revised. Deutsche Forschungsversion des Tests von L. M. Dunn \& L. M. Dunn von 1981. Universität Bamberg, Freie Universität Berlin.

Roßbach, H.-G. \& Weinert, S. (Hrsg.) (2008). Kindliche Kompetenzen im Elementarbereich: Förderbarkeit, Bedeutung und Messung. Bonn u.a.: Bundesministerium für Bildung und Forschung.

Scarborough, H. \& Dobrich, W. (1994). On the efficacy of reading to preschoolers. Developmental Review, 14, 3, S. 245-302.

Sénéchal, M. (2006). Testing the home literacy model: Parent involvement in kindergarten is differentially related to grade 4 reading comprehension, fluency, spelling, and reading for pleasure. Journal for the Scientific Study of Reading, 10, 1, S. 59-87.

Sénéchal, M. \& LeFevre, J. (2002). Parental involvement in the development of children's reading skill: A 5-year longitudinal study. Child Development, 73, 2, S. 445-460.

Sénéchal, M., LeFevre, J.-A., Thomas, E. \& Daley, K. (1998). Differential effects of home literacy experiences on the development of oral and written language. Reading Research Quarterly, 33, 1, S. 96-116.

Shanahan, T. \& Lonigan, C. J. (2010). The National Early Literacy Panel: A summary of the process and the report. Educational Researcher, 39, 4, S. 279-285.

Sigel, I. E. (1982). The relationship between parental distancing strategies and the child's cognitive behavior. In: Laosa, L. \& Sigel, I. E. (Hrsg.), Families as learning environments for children. New York: Plenum Press, S. 47-86.

Sigel, I. E. (1986). Early social experience and the development of representational competence. In W. Fowler (Ed.), Early experience and the development of competence. San Francisco: Jossey-Bass, S. 49-65.

Sigel, I. E., Stinson, E. T. \& Flaugher, J. (1991). Socialization of representational competence in the family: The distancing paradigm. In: Okagaki, L: \& Sternberg; R. J. (Hrsg.), Directors of development. Mahwah, NJ: Erlbaum, S. 121-144.

Snow, C. E. (1991). The theoretical basis form relationships between language and literacy in development. Journal of Research in Childhood Education, 6, 1, S. 5-10.

Snow, C. E. (1999). Social perspectives on the emergence of language. In: MacWhinney, B. (Hrsg.), The emergence of language. Mahwah, NJ: Erlbaum, S. 257-276.

Stanovich, K.E. \& West, R.F. (1989). Exposure to print and orthographic processing. Reading Research Quarterly, 24, 4, S. 402-433.

Storch, A. S. \& Whitehurst, G. J. (2002). Oral language and code-related precursors to reading: Evidence from a longitudinal structural model. Developmental Psychology, 38, 6, S. 934-947.

Totsika, V. \& Sylva, K. (2004). The Home Observation for Measurement of the Environment revisited. Child and Adolescent Mental Health, 9, 1, S. 25-35. 
Umek, L.M., Podlesek, A. \& Fekonja, U. (2005). Assessing the home literacy environment: Relationships to child language comprehension and expression. European Journal of Psychological Assessment, 21, 4, S. 271-281.

Van Kleeck, A., Gillam, R., Hamilton, L. \& McGrath, C. (1997). The relationship between middle-class parents' book-sharing discussion and their preschoolers' abstract language development. Journal of Speech-Language-Hearing Research, 40, 6, S. 1261-1271.

Van Steensel, R. (2006). Relations between socio-cultural factors, the home literacy environment and children's literacy development in the first years of primary education. Journal of Research in Reading, 29, 4, S. 367-382.

Wasik, B. (Ed.) (2004). Handbook of family literacy. Hillsdale, NJ: Erlbaum

Weinert, S., Ebert, S. \& Dubowy, M. (2010). Kompetenzen und soziale Disparitäten im Vorschulalter. Zeitschrift für Grundschulforschung, 3, 1, S. 32-45.

Weinert, S., Ebert, S., Lockl, K. \& Kuger, S. (2012). Disparitäten im Wortschatzerwerb: Zum Einfluss des Arbeitsgedächtnisses und der Anregungsqualität in Kindergarten und Familie auf den Erwerb lexikalischen Wissens. Unterrichtswissenschaft, 40, 1, S. 4-25.

Whitehurst, G. J. \& Lonigan, C. J. (1998). Child development and emergent literacy. Child Development, 69, 3, S. 848-872.

Whitehurst, G. J., Falco, F. L., Lonigan, C. J., Fischel, J. E., DeBaryshe, B. D., Valdez-Menchaca, M. C. \& Caulfield, M. (1988). Accelerating language development through book reading. Developmental Psychology, 24, 4, S. 552-559.

Whitehurst, G. J., Arnold, D. H., Epstein, J. N., Angell, A. L., Smith, M. \& Fischel, J. E. (1994a). A picture book reading intervention in daycare and home for children from low-income families. Developmental Psychology, 30, 5, S. 679-689.

Whitehurst, G. J., Epstein, J. N., Angell, A. L., Payne, A. C. Crone, D. A. \& Fischel, J. E. (1994b). Outcomes of an emergent literacy intervention in Head Start. Journal of Educational Psychology, 86, 4, S. 542-555.

Wygotski, L. S. (1969). Denken und Sprechen. Frankfurt a.M.: S. Fischer Verlag.

Eingereicht am/Submitted on: 26.03.2012

Angenommen am/Accepted on: 06.07.2012

Anschriften der Autorinnen und des Autors/Addresses of the authors:

Simone Lehrl, Diplom-Pädagogin (korrespondierende Autorin/corresponding author)

Otto-Friedrich-Universität Bamberg

BiKS-Forschergruppe

Luitpoldstraße 5

96052 Bamberg

Dr. Susanne Ebert

Otto-Friedrich-Universität Bamberg

Lehrstuhl Psychologie I

Markusplatz 3

96047 Bamberg

Prof. Dr. Hans-Günther Roßbach

Otto-Friedrich-Universität Bamberg

Lehrstuhl für Elementar- und Familienpädagogik

Markusstraße 8a

96047 Bamberg 
Prof. Dr. Sabine Weinert

Otto-Friedrich-Universität Bamberg

Lehrstuhl Psychologie I

Markusplatz 3

96047 Bamberg

Deutschland/Germany

E-Mail: simone.lehrl@uni-bamberg.de susanne.ebert@uni-bamberg.de hans-guenther.rossbach@uni-bamberg.de sabine.weinert@uni-bamberg.de 